\title{
Echocardiographic Profile of Hypertrophic Cardiomyopathy - A Single-Centre, Observational study
}

\author{
AKM Monwarul Islam¹, Dipal K Adhikary ${ }^{2}$, Shovan Rahman ${ }^{1}$, Mohsin Ahmed ${ }^{1}$, Md. Toufiqur \\ Rahman $^{3}$, Mohammad Ullah ${ }^{4}$, Abdullah AS Majumder ${ }^{5}$ \\ ${ }^{1}$ Department of Cardiology, NICVD, Dhaka, ${ }^{2}$ Department of Cardiology, Bangabandhu Sheikh Mujib \\ Medical University, Dhaka, ${ }^{3}$ Department of Cardiology, Col. Malek Medical College, Manikgonj, \\ ${ }^{4}$ Department of Cardiology, Sir Salimullah Medical College, Dhaka, ${ }^{5}$ Department of Cardiology, \\ Bangladesh Specialized Hospital, Dhaka
}

Key words:
Echocardiography,
Hypertrophic
Cardiomyopathy,
Phenotype,
Bangladesh.

\begin{abstract}
:
\end{abstract}
Background: Hypertrophic cardiomyopathy (HCM) is an autosomal dominant disease of left ventricular hypertrophy $(L V H)$. Phenotypic expression varies widely from subclinical hypertrophy to gross asymmetric septal hypertrophy causing left ventricular outflow tract (LVOT) obstruction. On top of genetic and phenotypic heterogeneity, the prevalence of different types of HCM may have geographical, as well as, ethnic variation.

Methods: This observational study was carried out during 2010 to 2020 to determine the echocardiographic profile of HCM in Bangladeshi population. All patients undergoing transthoracic echocardiography (TTE) in a private consultation centre of Dhaka, Bangladesh were included. HCM was defined as the presence of a maximal end-diastolic wall thickness of e"15 mm anywhere in the left ventricle $(L V)$, in the absence of another cause of hypertrophy in adults. HCM was further classified according to the pattern of myocardial hypertrophy and presence or absence of LVOT, or mid-left ventricular cavity obstruction.

Results: Out of 76 cases, non-obstructive HCM was the commonest type (65.8\%), followed by HCM causing LVOT obstruction (13.2\%), HCM causing mid-LV cavity obstruction (10.5\%), and the apical variety (10.5\%). Asymmetric septal hypertrophy (ASH) was found in $42.1 \%$, systolic anterior motion (SAM) of anterior mitral leaflet (AML) in $14.5 \%$, mitral regurgitation (MR) in $50 \%$, left ventricular systolic dysfunction in $5.3 \%$, and raised pulmonary artery systolic pressure (PASP) in $15.8 \%$ of cases. Maximum LV wall thickness $\geq 30 \mathrm{~mm}$ was found in 66 out of 76 cases.

Conclusion: The study highlights the clinically useful profile of HCM in Bangladeshi population based on conventional echocardiography. Further studies involving clinical, newer echocardiographic modalities and genetic analyses are warranted to discover the additional information in this ethnicity.

(Cardiovasc j 2021; 14(1): 5-11)

\section{Introduction:}

Hypertrophic cardiomyopathy (HCM) is characterized predominantly by left ventricular hypertrophy $(\mathrm{LVH})$ in the absence of another cardiac, systemic, or metabolic disease capable of producing the magnitude of hypertrophy evident in a given patient and for which a disease-causing sarcomere (or sarcomere-related) variant is identified, or genetic aetiology remains unresolved. ${ }^{1} \mathrm{HCM}$ is in fact a genetic disease with an autosomal dominant pattern of inheritance and variable expressivity. Mutation of sarcomere genes result in myofiber disarray and hypertrophy. Pattern of myocardial hypertrophy varies ranging from subtle preclinical hypertrophy to hypertrophy affecting single segment, multiple segments, apical

Address of Correspondence: Dr. AKM Monwarul Islam. Department of Cardiology, National Institute of Cardiovascular Diseases, Dhaka, Bangladesh. Email: drmonwarbd@yahoo.com.

- 2020 authors; llicensed and published by International Society of Cardiovascular Ultrasound, Bangladesh Chapter and Bangladesh Society of Geriatric Cardiology. This is an Open Access article distributed under the terms of the CC BY NC 4.0 (https://creativecommons.org/licenses/by-nc/4.0) 
segments to diffuse involvement of the left ventricle mimicking concentric hypertrophy. Involvement of right ventricle, i.e., biventricular involvement has also been reported. Besides variation in phenotypic expression, natural history of $\mathrm{HCM}$ also varies widely ranging from benign course to chronically symptomatic one to presentation with sudden death. Presence of left ventricular outflow tract (LVOT) or mid LV cavity obstruction and gross myocardial hypertrophy of $>30 \mathrm{~mm}$ may have prognostic significance. ${ }^{2-4}$ Conventionally, HCM is diagnosed and followed up by transthoracic echocardiography (TTE). But now-a-days, other imaging modalities like computed tomographic (CT) scanning, and specially, cardiac magnetic resonance (CMR) are playing increasing role in diagnosis and prognostication of HCM. Besides this, genotyping is contributing to the genetic basis of the disease, and thereby helping confirmation of the diagnosis and genetic counselling. Recognizing the genetic and phenotypic heterogeneity, the diagnostic criteria and classification of HCM are still evolving. Though probably more perfect, these classifications may not be so practical in day-to-day practice. Moreover, necessary technology and expertise may not be available widely. On the other hand, the prevalence of different types of HCM may have geographical, as well as, ethnic variation, e.g., the apical variety of HCM is commoner in Japan than in the western countries. ${ }^{5-7}$

In Bangladesh, the prevalence of different types of $\mathrm{HCM}$ is not known. Data regarding other characteristics of HCM are also sparse. So, the present study was planned to find out clinical types of HCM, along with their selected attributes.

\section{Study Methods:}

This was an observational study based on echocardiography to find out the pattern of HCM in Bangladesh. The study was carried out in a private consultation centre of Dhaka City during 2010 to 2020. All patients sent for TTE were included. Echocardiographic examination was carried out by Vivid 7 and Vivid E95 (GE Healthcare, 9900 Innovation Drive, Wauwatosa, WI 53226, USA) following the recommendations of the American Society of Echocardiography (ASE). HCM was defined by echocardiography as presence of a maximal end-diastolic wall thickness of $\geq 15 \mathrm{~mm}$ anywhere in the left ventricle, in the absence of another cause of hypertrophy in adults. ${ }^{1,8}$

Once diagnosed, HCM was further classified according to the pattern of myocardial hypertrophy and presence or absence of left ventricular outflow tract (LVOT), or mid-left ventricular cavity obstruction into 4 types: Diffuse non-obstructive, HOCM with LVOT obstruction, HOCM with midcavity obstruction, and the apical. Diffuse nonobstructive HCM was defined as basal septal thickness $\geq 15 \mathrm{~mm}$, or ratio of septal thickness to thickness

of inferior wall at midventricular level $>1.5$, no significantly raised LVOT or mid-cavity gradient. HOCM with LVOT obstruction, and HOCM with mid-cavity obstruction were defined as basal septal thickness $\geq 15 \mathrm{~mm}$, or ratio of septal thickness to thickness of inferior wall at midventricular level $>1.5$, PLUS LVOT or mid-cavity gradient $\geq 30$ $\mathrm{mmHg}$. Apical HCM was defined as apical thickness $\geq 15 \mathrm{~mm}$, or ratio of apical LV wall thicknesses to basal LV wall thicknesses $=1.3-1.5 .{ }^{9}$

\section{Results:}

The study includes $76 \mathrm{HCM}$ cases studied echocardiographically. Age range of the studied patients was 17 to 75 years, mean $51.75 \pm 12.50$ years. Out of 76 cases, 61 were male, and 15 were female. Non-obstructive HCM affecting multiple segments was the commonest type constituting $65.8 \%$ of cases, followed by HCM causing LVOT obstruction $13.2 \%$. HCM causing mid-LV cavity obstruction and the apical variety comprised of $10.5 \%$ each. So, non-obstructive HCM affecting multiple segments and the apical HCM, which is also non-obstructive, together constitutes 76.3\% of all cases. Similarly, the HCM causing LVOT and mid-ventricular obstruction together was $23.7 \%$. (Table I). Further analysis of other echocardiographic parameters revealed the distribution of features commonly encountered in HCM by echocardiography. Asymmetric septal hypertrophy (ASH) was found in $42.1 \%$, systolic anterior motion (SAM) of anterior mitral leaflet (AML) in $14.5 \%$, mitral regurgitation (MR) in 50\%, LV systolic dysfunction in $5.3 \%$, dilated left atrium (LA) in $75 \%$, and raised pulmonary artery systolic pressure (PASP) in $15.8 \%$ of cases. (Table II). Maximum LV 
wall thickness $\geq 30 \mathrm{~mm}$ was found in majority of cases, i.e., 66 out of 76 . (Table III).

\section{Table-I}

Clinical pattern of hypertrophic cardiomyopathy $(N=76)$.

\begin{tabular}{lcc}
\hline Type of HCM & Frequency & Percentage \\
\hline Diffuse, non-obstructive & 50 & 65.8 \\
HCM with LVOT obstruction & 10 & 13.2 \\
$\begin{array}{l}\text { HCM with mid-ventricular } \\
\text { obstruction }\end{array}$ & 8 & 10.5 \\
Apical & 8 & 10.5 \\
\hline Total & 76 & 100 \\
\hline
\end{tabular}

HCM, hypertrophic cardiomyopathy; LVOT, left ventricular outflow tract

\section{Table-II}

Echocardiographic findings in hypertrophic cardiomyopathy $(N=76)$.

\begin{tabular}{lcc}
\hline $\begin{array}{l}\text { Echocardiographic } \\
\text { parameter }\end{array}$ & Frequency & Percentage \\
\hline ASH & 32 & 42.1 \\
SAM of AML & 11 & 14.5 \\
MR & 38 & 50 \\
LV systolic dysfunction & 4 & 5.3 \\
Dilated LA & 57 & 75 \\
Raised PASP & 12 & 15.8 \\
\hline
\end{tabular}

ASH, asymmetric septal hypertrophy; SAM, systolic anterior motion; AML, anterior mitral leaflet; MR, mitral regurgitation; LV, left ventricle; LA, left atrium; PASP, pulmonary artery systolic pressure

\section{Table-III}

Distribution of the HCM cases by maximum LV wall thickness $(N=76)$.

\begin{tabular}{lccc}
\hline Type of HCM & $\begin{array}{c}\text { Maximum LV wall } \\
\text { thickness }<30 \mathrm{~mm}\end{array}$ & $\begin{array}{c}\text { Maximum LV wall } \\
\text { thickness } \geq 30 \mathrm{~mm}\end{array}$ & Total \\
\hline Diffuse, non-obstructive & 7 & 43 & 50 \\
HCM with LVOT obstruction & 1 & 9 & 10 \\
HCM with mid-ventricular obstruction & 0 & 8 & 8 \\
Apical & 2 & 6 & 8 \\
\hline Total & 10 & 66 & 76 \\
\hline
\end{tabular}

HCM, hypertrophic cardiomyopathy; LV, left ventricular; LVOT, left ventricular outflow tract

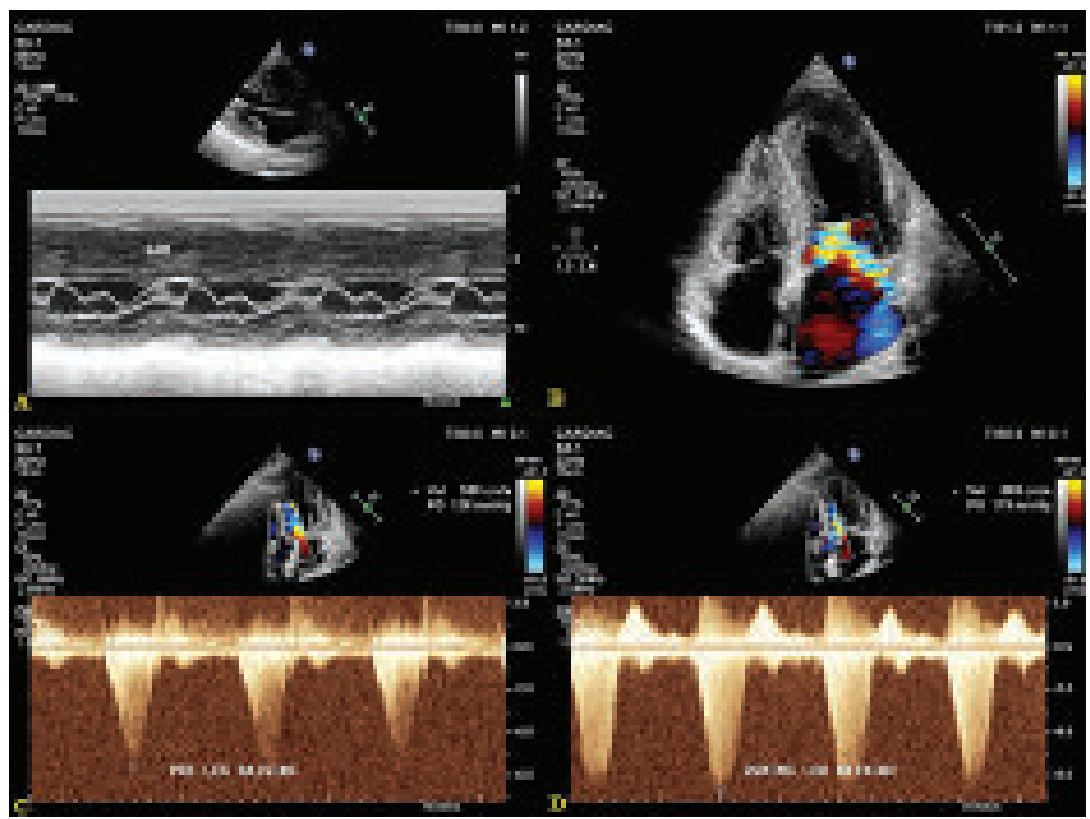

Fig .-1: Hypertrophic obstructive cardiomyopathy. Panel A, 2D-guided M-mode echocardiography showing asymmetric septal hypertrophy and systolic anterior motion (SAM) of anterior mitral leaflet (AML). Panel $B, 2 D$-guided colour Doppler imaging showing moderate eccentric mitral regurgitation caused by SAM of $A M L$. Panel C and D, 2D-and colour Doppler-guided spectral Doppler imaging showing raised left ventricular outflow tract gradient of $126 \mathrm{mmHg}$ at rest which increases to $179 \mathrm{mmHg}$ during leg raising. 


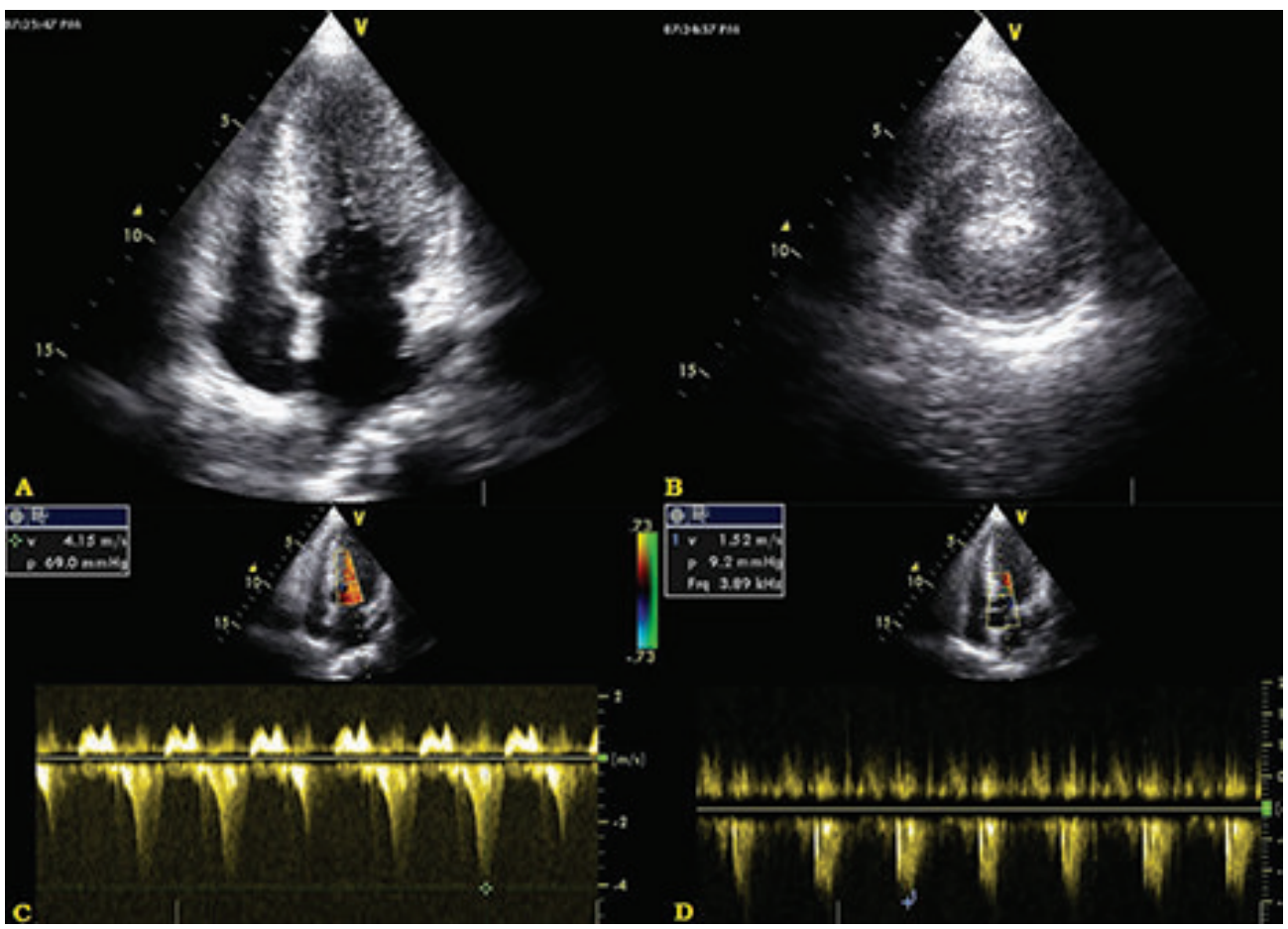

Fig.-2: Hypertrophic cardiomyopathy, mid-ventricular type. Panel A and B, 2D echocardiography apical long-axis view and apical short-axis view at the level of mid-ventricle respectively showing gross hypertrophy involving the mid segments of left ventricle almost obliterating the cavity in systole. Panel C, 2D-and colour Doppler-guided spectral Doppler imaging showing raised mid left ventricular cavity peak gradient of $69 \mathrm{mmHg}$ at rest. Panel D, 2D- and colour Doppler-guided spectral Doppler imaging showing normal left ventricular outflow tract peak gradient of $9.2 \mathrm{mmHg}$ at rest.
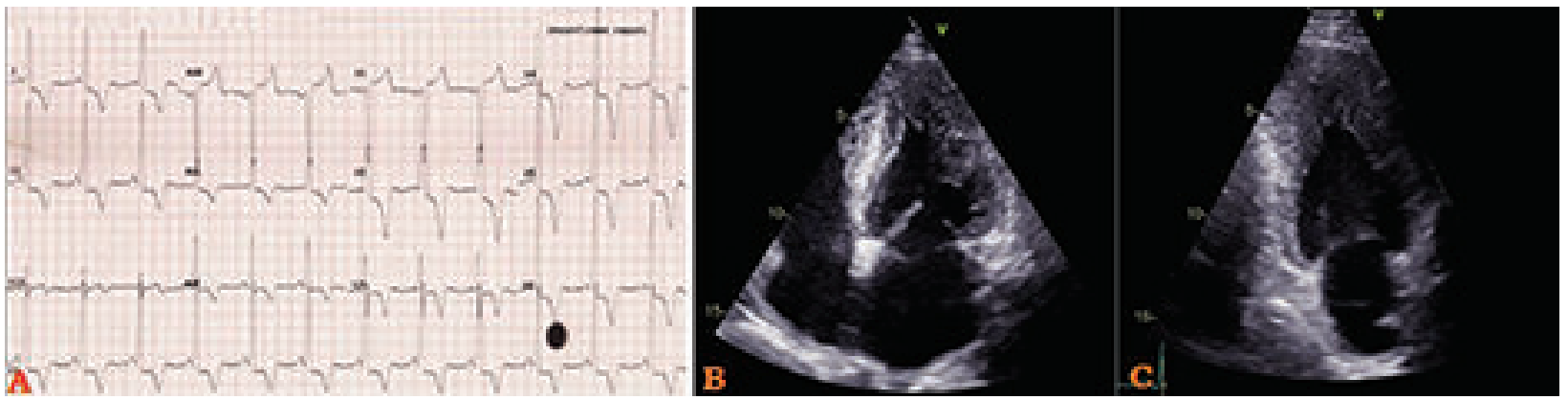

Fig.-3: Hypertrophic cardiomyopathy, apical variety. Panel A, electrocardiography showing deeply inverted $T$ waves in precordial leads. Panel B and C, 2D echocardiography apical 4-and 2-chamber view respectively showing gross hypertrophy involving the apical segments, as well as, the apex of left ventricle.

\section{Discussion:}

HCM is a disease of heterogeneity in terms of clinical presentation, natural history, morphology, and the genetic substrates that characterize inherited hypertrophic cardiomyopathy. ${ }^{10} \mathrm{Also}$, the classification of HCM is still in evolution. In the simplest form, only 2 phenotypes of HCM were identified: obstructive type (70\%) and nonobstructive type (30\%). ${ }^{11,12}$
HCM is popularly classified into 4 types: type I: hypertrophy involving the basal septum; type II: hypertrophy involving the whole septum; type III: hypertrophy involving septum, anterior, and anterolateral walls; type IV: LV apical hypertrophy. ${ }^{13}$ In between two, Wigle ED et al. classified HCM into three functional phenotypes: subaortic obstructive, midventricular obstructive and cavity obliterative. ${ }^{14}$ On the basis of septal 
contour and location and extent of hypertrophy, more complex classification has been proposed: reverse curvature, sigmoidal septum, neutral contour, apical form, mid-ventricular form. ${ }^{15}$ In 2006, the American Heart Association Working Group suggested that HCM should be defined genetically and not morphologically. ${ }^{16}$ Subsequently, the European Society of Cardiology Working Group on Myocardial and Pericardial Diseases recommended a morphological classification, the key point of this latter approach is that clinical evaluation of patients more often starts with the finding of a hypertrophied heart rather than a genetic mutation. ${ }^{17}$

In the present study, almost $3 / 4^{\text {th }}$ were nonobstructive HCM (classical and apical together), while only $1 / 4^{\text {th }}$ was obstructive type (LVOT and mid-cavity together). In the literature, presence of LVOT obstruction, either at rest or with provocation, varies widely from $25 \%$ to $75 \%$ of $\mathrm{HCM}$ cases. ${ }^{18,19}$ The lower prevalence of obstruction seen in this study may be due to the fact that, LVOT gradient was measured at rest only. In fact, most patients with HCM do not exhibit significant resting LVOT obstruction. ${ }^{19}$ On the other hand, midventricular obstructive HCM was found in $10.5 \%$ of cases. This figure is much higher than that of the reported prevalence of $1 \% .^{2}$ In this study, the proportion of apical HCM was $10.5 \%$. Among the different types of HCM, apical variety probably shows the highest ethnic variation in prevalence, accounting for up to $25 \%$ in Asian populations and $1 \%$ to $10 \%$ in non Asians. ${ }^{7,20,21}$ Recognizing the limitations of smaller sample size of the study, it can be concluded that, apical HCM is commoner in Bangladeshis. A study involving 34 Bangladeshi adults with HCM was carried out in the Bangabandhu Sheikh Mujib Medical University classified HCM into 3 types: asymmetric septal 56\%, concentric 29\%, apical 15\%. ${ }^{22}$ The study does not provide any information regarding the obstructive and non-obstructive types of HCM.

ASH was found in less than half of the HCM cases. ASH is defined by a septal-to-posterior diastolic wall thickness ratio $\geq 1.3$ (or $\geq 1.5$ in hypertensive patients). ${ }^{23}$ Presence of ASH is not diagnostic of HCM; sigmoid septum may commonly be confused with ASH. ASH may occur with or without LVOT obstruction. SAM of AML was found in $14.5 \%$ of
HCM patients which is lower than the reported prevalence of $30 \%$ to $60 \% .^{24}$ Like ASH, presence of SAM is not pathognomonic for HCM. SAM of AML may also occur in LV hypertrophy due to other causes, hyperdynamic states and hypovolaemia. Mitral regurgitation (MR) was noted in half of the HCM cases. In HCM, MR may result from LVOT obstruction or primary mitral valve leaflet abnormalities. MR associated with LVOT obstruction is typically mid-to-late systolic and eccentric with posteriorly or laterally directed jet. Three-fourths of the studied patients had dilated LA, a common association with $\mathrm{LVH}$ and $\mathrm{LV}$ diastolic dysfunction. Positive association has been described between left atrial size and sudden cardiac death. ${ }^{25,26}$ In 2014 ESC Guidelines on diagnosis and management of hypertrophic cardiomyopathy, left atrial diameter has been considered for risk stratification in HCM. ${ }^{2}$ Dilated LA also increases the risk of atrial fibrillation. Raised PASP and LV systolic dysfunction were found in $15.8 \%$ and $5.3 \%$ of cases.

In this study, out of 76 cases of HCM, 66 had maximum $\mathrm{LV}$ wall thickness $\geq 30 \mathrm{~mm}$. The severity and extent of LVH measured by transthoracic echocardiography are associated with the risk of sudden cardiac death in patients with HCM. Accordingly, extreme LV wall thicknesses of $\geq 30$ $\mathrm{mm}$ has been used for risk stratification. ${ }^{2-4}$

The study has got some important limitations. This was an echocardiography-based study, lacking relevant clinical information. Only, conventional echocardiography was done - parameters related to strain imaging, 3-dimentional echocardiography and stress echocardiography are lacking. LVOT gradient was measured at rest in general, so, some cases of obstructive HCM might be classified as non-obstructive type. Left ventricular contrast echocardiography was not used, hence, some cases of apical HCM might be missed. Genetic testing might detect subclinical HCM.

\section{Conclusion:}

HCM is a heterogenous type of cardiomyopathy. It appears to show significant ethnic and geographical diversity. The study highlights the clinically useful profile of HCM in Bangladeshi population based on conventional echocardiography. Further studies involving clinical, newer echocardiographic modalities and genetic 
analyses are warranted to discover the additional information in this ethnicity.

\section{Conflict of Interest - None.}

\section{References:}

1. Ommen SR, Mital S, Burke MA et al. 2020 AHA/ACC Guideline for the Diagnosis and Treatment of Patients with Hypertrophic Cardiomyopathy: A Report of the American College of Cardiology/American Heart Association Joint Committee on Clinical Practice Guidelines. Circulation. 2020 Dec 22; 142(25): e558e631. DOI: 10.1161/CIR.0000000000000937.

2. Authors/Task Force members, Elliott PM, Anastasakis A, Borger MA et al. 2014 ESC Guidelines on diagnosis and management of hypertrophic cardiomyopathy: the Task Force for the Diagnosis and Management of Hypertrophic Cardiomyopathy of the European Society of Cardiology (ESC). Eur Heart J. 2014 Oct 14; 35(39): 2733-2779. DOI: 10.1093/eurheartj/ehu284.

3. Autore C, Quarta G, Spirito P. Risk stratification and prevention of sudden death in hypertrophic cardiomyopathy. Curr Treat Options Cardiovasc Med. 2007 Dec; 9(6): 431-435. DOI: 10.1007/s11936-007-0037y.

4. Spirito P, Bellone P, Harris KM, Bernabo P, Bruzzi P, Maron BJ. Magnitude of left ventricular hypertrophy and risk of sudden death in hypertrophic cardiomyopathy. N Engl J Med. 2000 Jun 15; 342(24):1778-1785. DOI: 10.1056/NEJM200006153422403.

5. Eberly LA, Day SM, Ashley EA et al. Association of race with disease expression and clinical outcomes among patients with hypertrophic cardiomyopathy. JAMA Cardiol. 2020 Jan 1; 5(1): 83-91. DOI: 10.1001/ jamacardio.2019.4638.

6. Wells S, Rowin EJ, Bhatt V, Maron MS, Maron BJ. Association between race and clinical profile of patients referred for hypertrophic cardiomyopathy. Circulation. 2018 May 1;137(18):1973-1975. DOI: 10.1161/ CIRCULATIONAHA.117.032838.

7. Klarich KW, Attenhofer Jost CH, Binder J et al. Risk of death in long-term follow-up of patients with apical hypertrophic cardiomyopathy. Am J Cardiol. 2013 Jun 15; 111(12):1784-1791. DOI: 10.1016/j.amjcard.2013. 02.040 .

8. Gersh BJ, Maron BJ, Bonow RO et al.; American College of Cardiology Foundation/American Heart Association Task Force on Practice Guidelines. 2011 ACCF/AHA Guideline for the Diagnosis and Treatment of Hypertrophic Cardiomyopathy: a report of the American College of Cardiology Foundation/American Heart Association Task Force on Practice Guidelines. Developed in collaboration with the American Association for Thoracic Surgery, American Society of
Echocardiography, American Society of Nuclear Cardiology, Heart Failure Society of America, Heart Rhythm Society, Society for Cardiovascular Angiography and Interventions, and Society of Thoracic Surgeons. J Am Coll Cardiol. 2011 Dec 13; 58(25): e212-60. DOI: 10.1016/j.jacc.2011.06.011.

9. Baxi AJ, Restrepo CS, Vargas D, Marmol-Velez A, Ocazionez D, Murillo H. Hypertrophic cardiomyopathy from A to Z: Genetics, pathophysiology, imaging, and management. Radiographics. 2016 Mar-Apr; 36(2): 335354. DOI: 10.1148/rg.2016150137.

10. Maron BJ, Seidman CE, Ackerman MJ et al. How should hypertrophic cardiomyopathy be classified? What's in a name? Dilemmas in nomenclature characterizing hypertrophic cardiomyopathy and left ventricular hypertrophy. Circ Cardiovasc Genet. 2009 Feb; 2(1): 81-85; discussion 86. DOI: 10.1161/CIRCGENETICS. 108.788703 .

11. Braundwald E, Lambrew CT, Rockoff SD, Ross J Jr, Morrow AG. Idiopathic hypertrophic subaortic stenosis. I. A description of the disease based upon an analysis of 64 patients. Circulation. 1964 Nov; 30:Suppl 4: 3-119. DOI: $10.1161 / 01 . c i r .29 .5$ s4.iv-3.

12. Shapiro LM, McKenna WJ. Distribution of left ventricular hypertrophy in hypertrophic cardiomyopathy: a two-dimensional echocardiographic study. J Am Coll Cardiol. 1983 Sep; 2(3): 437-444. DOI: 10.1016/s0735-1097(83)80269-1.

13. Maron BJ, Gottdiener JS, Epstein SE. Patterns and significance of distribution of left ventricular hypertrophy in hypertrophic cardiomyopathy. A wide angle, two-dimensional echocardiographic study of 125 patients. Am J Cardiol. 1981 Sep; 48(3): 418-428. DOI: 10.1016/0002-9149(81)90068-0.

14. Wigle ED, Sasson Z, Henderson MA et al. Hypertrophic cardiomyopathy. The importance of the site and the extent of hypertrophy. A review. Prog Cardiovasc Dis. 1985 Jul-Aug; 28(1): 1-83. DOI: 10.1016/00330620(85)90024-6.

15. Syed IS, Ommen SR, Breen JF, Tajik AJ. Hypertrophic cardiomyopathy: identification of morphological subtypes by echocardiography and cardiac magnetic resonance imaging. JACC Cardiovasc Imaging. 2008 May; 1(3): 377-379. DOI: 10.1016/j.jcmg.2008.02.008.

16. Maron BJ, Towbin JA, Thiene G et al.; American Heart Association; Council on Clinical Cardiology, Heart Failure and Transplantation Committee; Quality of Care and Outcomes Research and Functional Genomics and Translational Biology Interdisciplinary Working Groups; Council on Epidemiology and Prevention. Contemporary definitions and classification of the cardiomyopathies: an American Heart Association Scientific Statement from the Council on Clinical Cardiology, Heart Failure and Transplantation Committee; Quality of Care and Outcomes Research and Functional Genomics and Translational Biology Interdisciplinary Working Groups; 
and Council on Epidemiology and Prevention. Circulation. 2006 Apr 11;113(14):1807-1816. DOI: 10.1161/CIRCULATIONAHA. 106.174287.

17. Elliott P, Andersson B, Arbustini E et al. Classification of the cardiomyopathies: a position statement from the European Society of Cardiology Working Group on Myocardial and Pericardial Diseases. Eur Heart J. 2008 Jan; 29(2): 270-276. DOI: 10.1093/eurheartj/ehm342.

18. Maron MS, Olivotto I, Zenovich AG et al. Hypertrophic cardiomyopathy is predominantly a disease of left ventricular outflow tract obstruction. Circulation. 2006 Nov 21; 114(21): 2232-2239. DOI: 10.1161/ CIRCULATIONAHA.106.644682.

19. D'Andrea A, Caso P, Cuomo S et al. Prognostic value of intra-left ventricular electromechanical asynchrony in patients with mild hypertrophic cardiomyopathy compared with power athletes. Br J Sports Med. 2006 Mar; 40(3): 244-250. DOI: 10.1136/bjsm.2005.022194.

20. Parato VM, Olivotto I, Maron MS, Nanda NC, Pandian NG. Left ventricular apex involvement in hypertrophic cardiomyopathy. Echocardiography. 2015 Oct; 32(10): 1575-1580. DOI: 10.1111/echo.12986.

21. Maron MS, Maron BJ, Harrigan C et al. Hypertrophic cardiomyopathy phenotype revisited after 50 years with cardiovascular magnetic resonance. J Am Coll Cardiol.
2009 Jul 14; 54(3): 220-228. DOI: 10.1016/j.jacc. 2009.05.006.

22. Masum MM, Shahrear R, Yeasmin ZA, Nishat L, Banu LA. Evaluation of LVEF with changing left ventricular morphology in hypertrophic cardiomyopathy. University Heart Journal 2019; 15(1): 12-5.

23. Williams LK, Frenneaux MP, Steeds RP. Echocardiography in hypertrophic cardiomyopathy diagnosis, prognosis, and role in management. Eur $J$ Echocardiogr. 2009 Dec;10(8):iii9-14. DOI: 10.1093/ ejechocard/jep157.

24. Parato VM, Antoncecchi V, Sozzi F et al.; Italian Chapter of ISCU. Echocardiographic diagnosis of the different phenotypes of hypertrophic cardiomyopathy. Cardiovasc Ultrasound. 2016 Aug 12;14(1):30. DOI: 10.1186/s12947-016-0072-5.

25. O'Mahony C, Jichi F, Pavlou M et al.; Hypertrophic Cardiomyopathy Outcomes Investigators. A novel clinical risk prediction model for sudden cardiac death in hypertrophic cardiomyopathy (HCM risk-SCD). Eur Heart J. 2014 Aug 7; 35(30): 2010-20. DOI: 10.1093/ eurheartj/eht439.

26. Spirito P, Autore C, Rapezzi C et al. Syncope and risk of sudden death in hypertrophic cardiomyopathy. Circulation. 2009 Apr 7; 119(13): 1703-1710. DOI: 10.1161/CIRCULATIONAHA.108.798314. 